\title{
Microcomputer-Assisted Selection of Optimum Timing for Titrant Addition in Automated Potentiometric Precipitation Titration
}

\author{
Shuko FujIEDA and Nobue Nishi ${ }^{\dagger}$ \\ Department of Chemistry, Faculty of Science, Ochanomizu University, Otsuka, Tokyo 112, Japan
}

\begin{abstract}
An intelligent potentiometric titrator is described which can carry out automatic precipitation titrations under optimum conditions and minimum time. The buret tip is not immersed in the titrand solution as is usually the case, but is held above the solution so as to prevent the titrant from leaking and reacting with the titrand solution during waiting intervals. The titrant is added drop by drop with a motor-driven buret. After each drop of titrant has been added, the buret is stopped and values of the potential difference obtained with predetermined short time intervals (e.g. $1 \mathrm{~s}$ ) are stored in a microcomputer. From the convergence of several successive values, the microcomputer determines whether there has been a substantial attainment of the precipitation equilibrium, stores the final value in the memory and sends a control signal to the buret to resume titrant addition. These operations are repeated until the equivalence point is passed. The exact equivalence point could be evaluated by any common method. Particularly, Gran's plot method is preferred. The appropriateness of the system, including hardware and software, has been demonstrated by titration of silver ions with chloride, which gave very reasonable results.
\end{abstract}

Keywords Microcomputer control, automatic titrator, optimization, potentiometry, precipitation titration

The complete formation of a precipitate after the addition of a small amount of a titrant is timeconsuming in general precipitation titrations. Moreover, the time necessary for attaining equilibrium differs according to the titration process and may depend on the nature of the reaction and experimental conditions. An automatic titrator of a popular type allows the operator to select such modes of titrant addition as the delivery rate of the titrant and the waiting interval for equilibrium prior to starting titration. However, some experiences are prerequisite for the selection of such parameters, since the progress of each titration may be affected by experimental conditions as the temperature, concentration, and stirring rate of the titrand or the solution to be titrated. Therefore, if the timing of titrant addition is selected uniformly, titration may proceed without attaining the precipitation equilibrium or sometimes with a waste of waiting time.

In most precipitation titrations which generally take time, the titrant should be prevented from leaking from the buret tip and mixing with the titrand during the intervals waiting for the complete formation of a precipitate. In a previous study' a buret tip was placed

Present address: Editorial Department, Tokyo Kagaku Dozin Co. Ltd., Sengoku, Bunkyo, Tokyo 112 above the titrand and the titrant was added drop by drop. The titrant addition was controlled by a microcomputer-aided system in which the fall of titrant drops was detected by a set of a light-emitting diode (LED) and a phototransistor to stop an electrically driven buret.

In the present study titrant solutions were also added in drops. Precipitation titration of silver with chloride was followed potentiometrically with a silver electrode against a saturated calomel electrode (SCE). Each time a titrant drop was added, the variation of the potential difference was monitored so as to automatically select the optimum timing of the following addition of the titrant. Upon confirming that no significant drift in the potential difference took place, the next addition of a drop followed. This process was repeated until the equivalence point was obtained. The whole assembled system was controlled by a program in BASIC. Upon each addition of a drop, the potentiometric readings converged to an equilibrium point, which could be effectively estimated without delay. To locate the point, some practical methods were compared so as to judge the equilibrium and to obtain the convergence value. Then, the equivalence point was estimated both on- and off-line by a common 2nd-derivative method, Gran's plot method ${ }^{2}$, Lagrange's interpolation method and a calculation of the inflection point of a 3rd-order 
equation obtained from the method of least squares. For saving both titration time and titrant volume, the anticipation of the equivalence point was also examined by Gran's plot technique, in which the volumes of titrant drops may differ from each other.

\section{Experimental}

\section{Reagents}

Guaranteed grade reagents of silver nitrate and sodium chloride from Wako Chemicals Co. (Tokyo) were used without further purification. Stock solutions of about $0.05 \mathrm{M}$ were prepared by dissolving weighed solid reagents in deionized water which was distilled before use.

\section{Apparatus}

A schematic block diagram of the whole system (assembled) is shown in Fig. 1. A microcomputer (M5, SORD, Tokyo) working with an 8-bit central processing unit (Z-80A, Zilog) was used for acquiring data via two digital voltmeters which indicate the titrant volume and the potential difference. It was also used to send signals to control the whole system, select the optimum timing of titrant addition, and calculate the equivalence point. In an extension box of the microcomputer, interface circuits constructed by the authors were included, as well as the ROM of a floating-point BASIC and 32-KB RAM boards. All of the control signals and data acquisition were carried out via two programmable peripheral interfaces (PPI) (i8255, Intel). A detailed description of the PPI was omitted, because PPI is now commonly used.

A glass syringe buret (E274-10, Metrohm) of $10 \mathrm{~cm}^{3}$

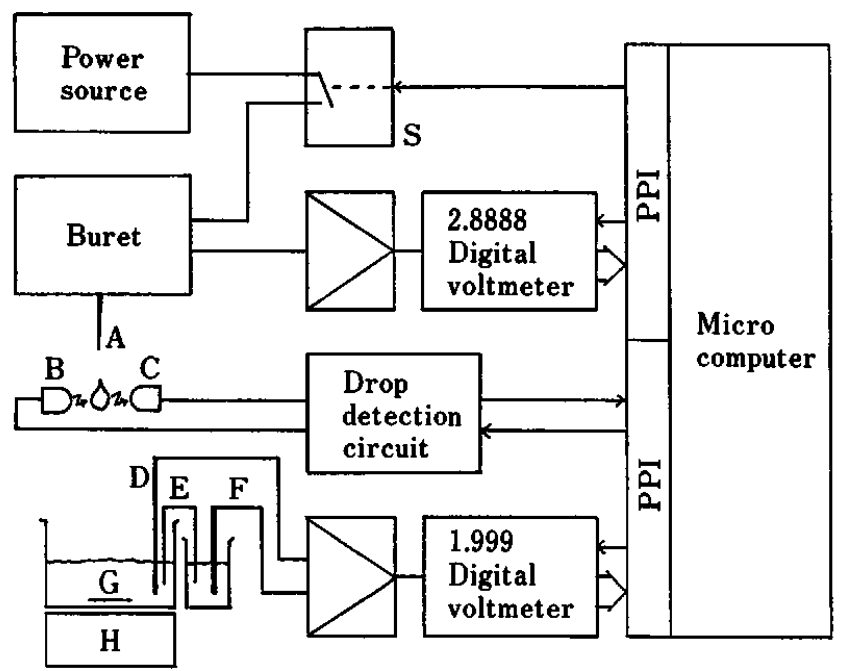

Fig. 1 Schematic diagram of the whole system. A, buret tip; B, LED; C, phototransistor; D, indicator electrode; E, salt bridge; F, SCE; G, iron bar; H, magnetic stirrer; S, solid state relay; PPI, programmable peripheral interface. (full scale) was modified so that it could be driven at appropriate speeds with a synchronous motor and gear combinations. A small glass syringe of $1 \mathrm{~cm}^{3}$ (full scale; HS-1b, Toa Electronic Co., Tokyo) was partly used. The start/stop switching of the buret was carried out by the on-off control of the power supply via a solid-state relay (SSR) (TSS1G-41, Toshiba, Tokyo) which was controlled by TTL level signals sent from the microcomputer. The switching delay was negligibly small. The piston of the syringe buret was linked to a 10-turn precision potentiometer $(100 \mathrm{k} \Omega$ in full scale with a linearity error of less than $\pm 0.3 \%$ ), to which a stabilized power source (521C, Metronix, Tokyo) of $2 \mathrm{~V}$ (full scale) was applied so that the volume of the titrant released from the buret would correspond to the voltage. The voltages were stored in the microcomputer via a digital voltmeter (DVM) of $\pm 2.8888 \mathrm{~V}$ (full scale; SC-53, Thinky, Tokyo), to which the input voltage was, if necessary, accordingly multiplied using an operational amplifier circuit. The titrant volume $v$ was obtained as the difference of two readings.

A set of the LED and the phototransistor, the drop detection circuit, and the potentiometric titration cell were constructed in the manner previously reported. ${ }^{1}$

A silver rod of a $1.3 \mathrm{~mm}$ diameter was used as an indicator electrode, and a popular type of SCE as a reference electrode. The observed potential difference $V$ was stored every second in the microcomputer via a DVM of $\pm 1.999 \mathrm{~V}$ (full scale; SC-51 Thinky, Tokyo) with a nominal input impedance larger than $1000 \mathrm{M} \Omega$, to which the input voltage range was also, if necessary, adjusted by operational amplifier circuits. In the present study, for simplicity a temperature-regulated bath was not used. However, the potentiometric cell was protected against heat from the stirrer motor by using a thermal insulation board. The potentiometric titration procedure was conducted at $25.0 \pm 0.1^{\circ} \mathrm{C}$. Two DVMs were calibrated by a Weston standard chemical cell.

\section{Software}

Programs in BASIC used for the automatic titration were divided into three functional parts: (A) Management of the signals used to control the entire hardware system and on-line data acquisitions of $v$ and $V$. (B) Automatic detection of the complete precipitate formation in each addition of a drop. (C) On- and off-line calculations of the equivalence point. Three parts of the programs were combined so that the entire hardware system could take part effectively. As for (B) and $(C)$, several methods were compared.

Regarding (A), programs included control words to two PPIs, signals for SSR to start and stop the power supply to the motor-driven buret, acquiring a latched set signal in a flip-flop circuit for detecting the fall of each titrant drop and putting out a reset signal to the circuit, aquisition of two kinds of voltage corresponding to $v$ and $V$, and control signals for each operation. $A$ built-in clock of the microcomputer was used for sampling $V$ every second. 
Table 1 Threshold values given for judging equilibrium, the required time and the estimated potential difference

\begin{tabular}{clcc}
\hline Method & \multicolumn{1}{c}{ Threshold value } & Time/s & $E / \mathrm{V}$ \\
\hline B1 & slope less than $1 \times 10^{-6}$ & 69.5 & $1.266_{7}$ \\
B2 & less than $\pm 1^{\mathrm{a}}$ & 52.5 & $1.267_{0}$ \\
B3 & less than $\pm 2^{\mathrm{a}}$ & 16.5 & $1.271_{2}$ \\
B4 & less than $2 \times 10^{-5}$ & 14.5 & $1.272_{2}$ \\
B5 & zero & 20.5 & $1.270_{0}$ \\
\hline
\end{tabular}

a. Shown on LSD.

Regarding (B), upon every addition of a drop, the progression of $V$ acquired in (A) was monitored in order to detect the attainment of precipitation equilibrium. The following five methods (Table 1) were compared for a rapid and reliable decision concerning the attainment of equilibrium. $V$ was determined at the attainment of $v$ as an observed potential difference $E$ (volts). In typical runs, the $V^{\prime}$ s in 10 or 20 points were used for each calculation.

(BI) Method of least squares. After every acquisition of a set of $V$ and time $t$, the slope of a straight line was calculated by a least-square approximation. When the obtained slope fell to within a given threshold value, precipitation equilibrium was considered to have been attained.

(B2) Difference of adjacent means. When the indicated number of means of $V$ fell continuously within the threshold value, their mean was used as $E$ at $v$. In typical runs the indicated number was set to 3 , and the threshold value set to \pm 1 on least significant digit (LSD) from the specific error of the DVM used.

(B3) Difference between maximum and minimum values. When the difference in $V$ was within the threshold value indicated, detection was completed. The range was then set to \pm 2 on the LSD.

(B4) Summation of the squares of difference. The squares of difference between adjacent $V$ 's were summed by the indicated numbers. If the total was within the threshold value, precipitate formation was considered to be completed at $v$.

(B5) (B4) with sign. If the measuring system is noisy or acquires fluctuating data, it took time, even in the equilibrium state, to make a decision on concerning the completion of precipitate formation. Therefore, a sign $(+$ or -$)$ was included in the summation, resulting in a zero in the undrifted state.

In the 3rd part (C), the following 4 popular techniques were used for estimating the equivalence point from the $E$ and $v$ obtained in (B).

(CI) Newtonian difference method. ${ }^{3}$ From 4 sets of $E$ and $v, v$ at which the 2 nd derivative of $E$ was zero was estimated as being the equivalence point.

(C2) Gran's plot method. ${ }^{2}$ If a substance $S$ to be titrated of a volume $v_{0}$ and an unknown concentration $C_{\mathrm{So}}$ is titrated with a titrant of concentration $C_{\mathrm{T} 0}, E$ in the potentiometric titration cell to which the titrant of volume $v$ was added is expressed by Eq. (1). The concentrations were used in place of the activities.

$$
E=E^{0}+k_{1} \log C_{\mathrm{S}}
$$

where $E^{0}$ is the standard potential $E_{\mathrm{Ag}^{+} / \mathrm{Ag}_{\mathrm{g}}}^{0}-E_{\mathrm{SCE}}, k_{1}$ is $2.303 R T / F$, and $C_{\mathrm{s}}$ is the concentration of $\mathrm{S}$ left unreacted and equals $\left(C_{\mathrm{s} 0} v_{0}-C_{\mathrm{T} 0} v\right) /\left(v_{0}+v\right)$. From Eq. (1), Eq. (2) is obtained:

$$
\left(v_{0}+v\right) 10^{E / k_{1}}=k_{2}-k_{3} v
$$

where $k_{2}=10^{E_{0} / k_{1}} C_{\mathrm{S} 0} v_{0}$ and $k_{3}=10^{E_{0} / k_{1}} C_{\mathrm{TO}}$. A plot of the left-hand side of Eq. (2) against $v$ may give a straight line. The point of intersection of the line with the axis of the abscissa indicates the titrant volume to be added at the equivalence point, so that $C_{\mathrm{So}}$ is calculated from $C_{\mathrm{To}} v / v_{0}$. At a constant temperature, during every aquisition of a set of $E$ and $v$ to the microcomputer, the equivalence point was anticipated from the linear leastsquare calculation by using $n$ sets of $\left(v_{i}, E_{i}\right)$ including the latest data.

(C3) Least square approximation for a cubic equation.

In the vicinity of an equivalence point, a titration curve can be expressed by a cubic equation: $f(x)=a x^{3}+b x^{2}$ $+c x+d$. The equivalence point can be calculated from $x=-b / 3 a$ at which the 2 nd derivative of the expression is zero. The cubic equation was obtained from the latest sets of $\left(x_{i}, y_{i}\right)$, where $i=1,2, \cdots, n$; that is equivalent to $\left(v_{i}, E_{i}\right)$, where $i=1,2, \cdots, n$ and $n$ is larger than 4. Simultaneous equations of 4 dimensions were obtained from the summation of $\left[f\left(x_{i}\right)-y_{i}\right]^{2}$. Determinant equations were dissolved by the Gaussian elimination method. An equal size of titrant drops was not required.

(C4) Lagrange's interpolation method. From 4 sets of $\left(x_{i}, y_{i}\right)(i=1,2,3$ and 4), which corresponded to $v$ and $E$, respectively, a cubic recurrence formula, $f(x)=a x^{3}+b x^{2}$ $+\mathrm{cx}+d$, was obtained. The equivalence point, at which the 2nd derivative is zero, may be estimated as $x$ equals to $-b / 3 a$, provided that coefficients $a$ and $b$ of $x^{3}$ and $x^{2}$, respectively, are calculated. When the maximum value of the first derivative exists between $y_{2}$ and $y_{3}$ the following expressions may be obtained by rearranging the recurrence formula:

$$
\begin{aligned}
a= & \left(y_{1} / A_{1}\right)+\left(y_{2} / A_{2}\right)+\left(y_{3} / A_{3}\right)+\left(y_{4} / A_{4}\right), \\
b= & -\left\{\left[y_{1}\left(x_{2}+x_{3}+x_{4}\right) / A_{1}\right]\right. \\
& +\left[y_{2}\left(x_{1}+x_{3}+x_{4}\right) / A_{2}\right] \\
& +\left[y_{3}\left(x_{1}+x_{2}+x_{4}\right) / A_{3}\right] \\
& \left.+\left[y_{4}\left(x_{1}+x_{2}+x_{3}\right) / A_{4}\right]\right\} \\
A_{1}= & \left(x_{1}-x_{2}\right)\left(x_{1}-x_{3}\right)\left(x_{1}-x_{4}\right), \\
A_{2}= & \left(x_{2}-x_{1}\right)\left(x_{2}-x_{3}\right)\left(x_{2}-x_{4}\right), \\
A_{3}= & \left(x_{3}-x_{1}\right)\left(x_{3}-x_{2}\right)\left(x_{3}-x_{4}\right),
\end{aligned}
$$

and

$$
A_{4}=\left(x_{4}-x_{1}\right)\left(x_{4}-x_{2}\right)\left(x_{4}-x_{3}\right) .
$$




\section{Procedure}

A titrand solution was taken in a glass beaker of 100 $\mathrm{cm}^{3}$ capacity with a microsyringe; a potentiometric titration cell was set up in a common manner. The titrant was placed in a motor-driven syringe buret. The initial condition and initialization signals were delivered to every interface device from the microcomputer, the whole system being in the stand-by state. The initial titrant volume was acquired from DVM via the potentiometer connected to the syringe piston.

Titration was started by driving the electric buret to which a control signal was given via SSR from the microcomputer. When a titrant drop fell from buret tip, a set of LED-phototransistor detected the passage to give a set signal to a flip-flop device in the dropdetection circuit (Fig. 1). When the microcomputer found the flag, a stop signal was sent to the motordriven buret via the SSR. The titrant volume was acquired again and a reset signal was sent to the flipflop circuit. Then, the acquisition of $V$ was started so as to detect the completion of precipitation, which was determined from the progress of $V$ vs. $t$ by using a program in (B). After estimating $E$ at $v$, the computer resumed the addition of drops, and repeated the same procedure until the equivalence point was obtained by the program specified in (C). If necessary, a command for successively adding several drops through instructions to the titrator may be included in the program.

The whole titration procedure was executed by automatically selecting the optimum timing of titrant addition. The progress of titration was displayed on a monitor screen and two DVMs which measured $v$ and V.

\section{Results and Discussion}

In a previous study ${ }^{1}$, the titrant volume was obtained as the product of the number of drops added and the drop size. However, constancy in the size of each drops was influenced by the cleanliness of the buret tip. It was also troublesome to measure the size of a drop. In the present work, the relation between the titrant volume released from the syringe buret and the resistance of the potentiometer was examined at six points. The mean of each 100 data was $9.640 \pm 0.0024$ $\mathrm{k} \Omega \mathrm{cm}^{-3}$. The final error of $v$ stored in the microcomputer via the DVM corresponded to less than \pm 0.25 digit. The delivery speed of the titrant was not required to be constant.

For demonstration, a sample solution containing silver ions was titrated with chloride ions. After every addition of a titrant drop, the potential difference of the titrand solution measured every second was acquired into the microcomputer. In Fig. 2, (A) represents a typical potentiometric titration curve or plot of $E v s . v$. After each addition of a titrant drop, (B) in Fig. 2 demonstrates the detail of $V$ vs. $t$, from which the precipitation equilibrium may be attained in due time.

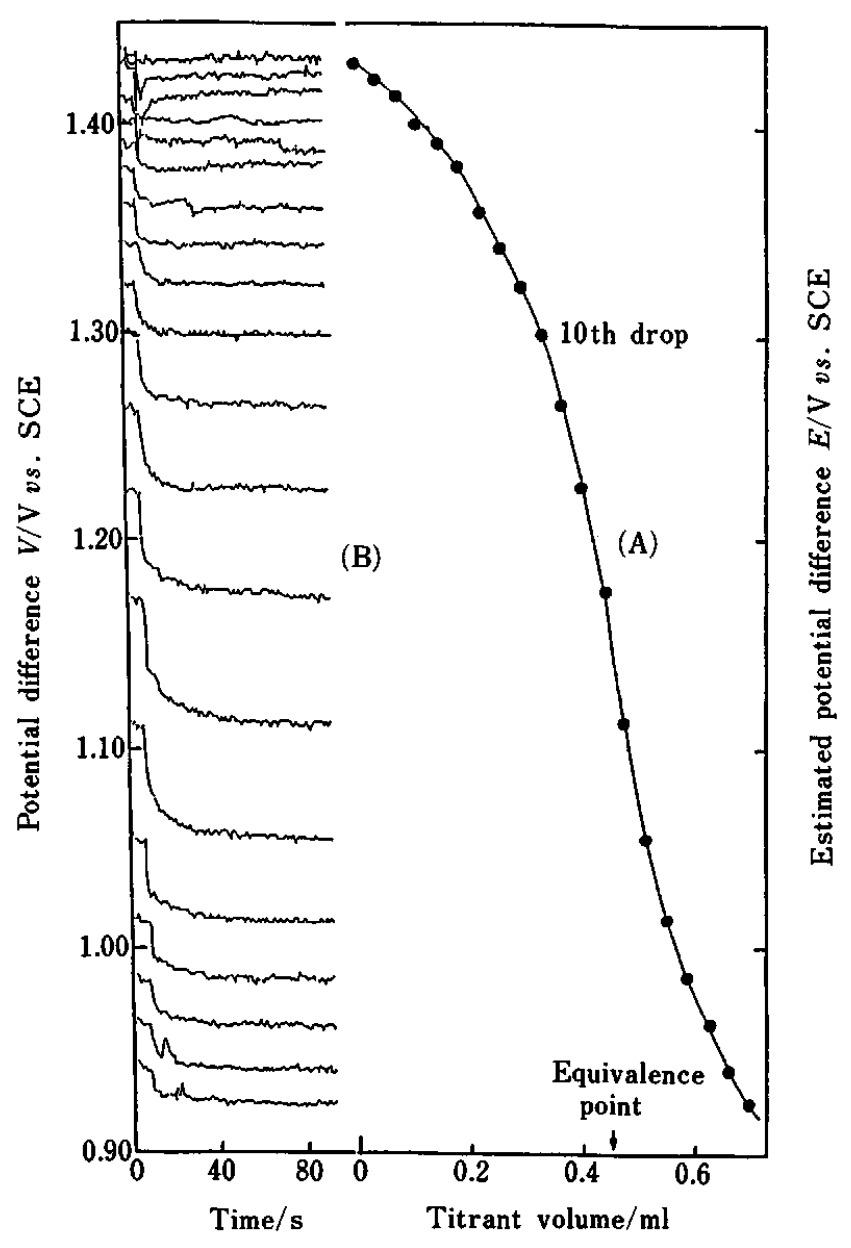

Fig. 2 Potentiometric titration of silver ions with chloride ions and a corresponding plot of the potential difference $v s$. the time after each addition of a titrant drop.

Near the equivalence point, it took a longer time before attaining equilibrium. Thus, when the attainment of equilibrium was detected and confirmed, the next drop was added. In this manner titrations may be carried out in a minimum amount of time and under optimum conditions. Therefore, an effective method for detecting the levelled state of a trail was necessary for automatic on-line treating. For this purpose, five calculation methods (B1 to B5 in Table 1) were examined. Ten data points were used for calculating the summation of squares. When the drift of $V$ becomes within the given threshold value, a large jump in the value should appear in the summation of squares for the detection of equilibrium. In the case of the 10th titrant drop (Figs. 2 and $3(\mathrm{~A})$ ), the behaviors of the summed squares obtained by B1, B4 and B5 are plotted against $t$ in Fig. 3 (B), as (1) (solid line), (3) (solid line), and (4) (dotted line), respectively. The use of 20 data points (2) (dotted line) in Fig. 3 (B) was compared with that of 10 data points by B1. However, no essential difference was found. The threshold value in the potential difference considered as being levelled, the 
Table 2 Influence of the decision method on the equivalence point obtained ${ }^{\text {a }}$

\begin{tabular}{cccccc}
\hline Taken & B1 & B2 & B3 & B4 & B5 \\
\hline 216.0 & $215.9 \pm 0.1$ & $216.2 \pm 0.1$ & $216.1 \pm 0.1$ & $216.8 \pm 0.1$ & $216.6 \pm 0.1$ \\
285.6 & $286.0 \pm 0.4$ & $285.2 \pm 0.4$ & $285.4 \pm 0.4$ & $285.5 \pm 0.4$ & $285.5 \pm 0.4$ \\
287.4 & $287.1 \pm 0.4$ & $287.3 \pm 0.4$ & $288.0 \pm 0.4$ & $288.1 \pm 0.4$ & $287.7 \pm 0.4$ \\
288.5 & $288.5 \pm 0.5$ & $288.7 \pm 0.5$ & $288.6 \pm 0.5$ & $289.8 \pm 0.5$ & $289.6 \pm 0.4$ \\
432.1 & $432.2 \pm 0.2$ & $431.7 \pm 0.2$ & $432.1 \pm 0.2$ & $432.3 \pm 0.2$ & $432.4 \pm 0.2$ \\
571.2 & $571.3 \pm 0.8$ & $571.7 \pm 0.8$ & $571.9 \pm 0.8$ & $571.6 \pm 0.8$ & $571.8 \pm 0.8$ \\
574.8 & $574.3 \pm 0.7$ & $574.4 \pm 0.7$ & $574.4 \pm 0.7$ & $574.2 \pm 0.7$ & $574.4 \pm 0.7$ \\
\hline
\end{tabular}

a. Found $\mathrm{Ag}^{+}$in $\mu \mathrm{mol}$ against taken. Figures following \pm show mean range of ambiguity in each judgment of equilibrium.

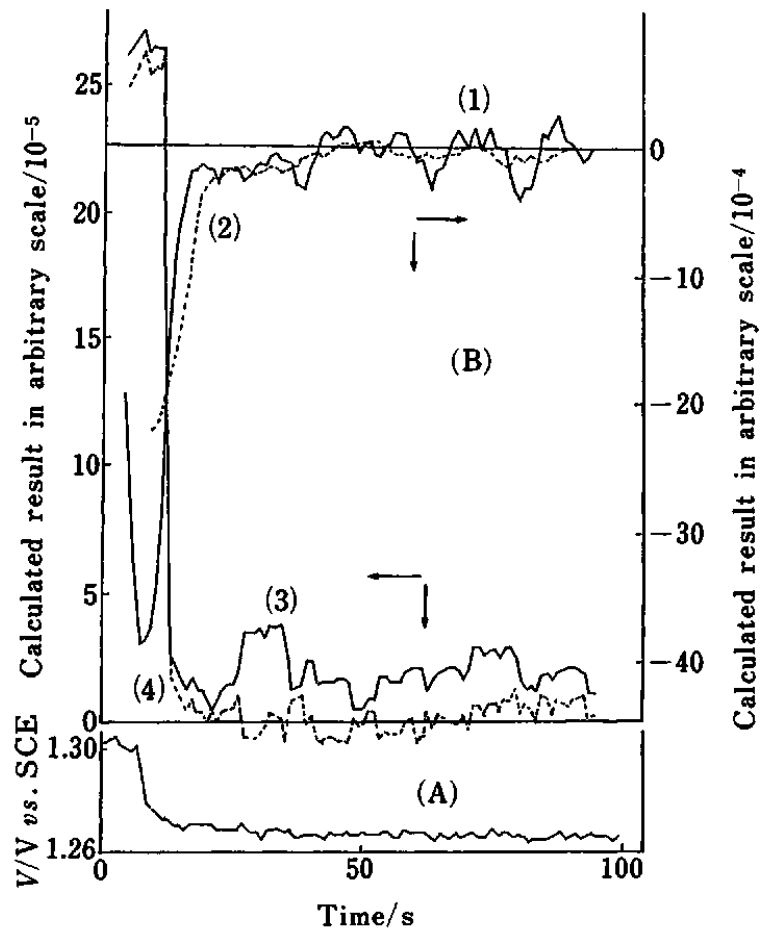

Fig. 3 Calculation for the judgment of precipitation equilibrium at the 10th drop (Fig. 2). The ordinate is shown in an arbitrary scale for a comparison.

time necessary for a decision, and the estimated $E$ are compared in Table 1 using data acquired at the 10th drop. The method of the least squares (B1) was popular (in general), but required time for calculations. In the present report, a subroutine in machine language and a BASIC compiler were, for simplicity, not employed. The mean of $E$, estimated using the five methods, was $1.269_{4} \mathrm{~V}$ with a standard deviation of $0.0025 \mathrm{~V}(0.19 \%)$.

The equivalence points of 13 samples were estimated by the Newtonian difference method $(\mathrm{Cl})$, the results being shown in Table 2 as the mean of 3 or 4 runs. Errors are not shown as the ratio of moles of $\mathrm{Ag}^{+}$found to that taken, since no significant difference was found among the decision methods. In several examples, 20 data points were also used for calculating the equiva-
Table 3 Comparison of the equivalence point estimated by 4 methods $^{\mathrm{a}}$

\begin{tabular}{|c|c|c|c|c|c|}
\hline \multirow{2}{*}{ Taken $^{b}$} & \multirow{2}{*}{$\mathrm{Cl}$} & \multicolumn{2}{|c|}{$\mathrm{C} 2$} & \multirow{2}{*}{ C3 } & \multirow{2}{*}{$\mathrm{C} 4$} \\
\hline & & 8 data & 6 data & & \\
\hline 116.9 & 0.1 & -0.2 & -0.5 & 0.1 & 0.1 \\
\hline $222.4^{\mathrm{c}}$ & -1.1 & 0.07 & -0.4 & -0.5 & -1.3 \\
\hline $222.4^{\mathrm{c}}$ & -5.2 & 0.6 & 0.2 & 0.7 & -6.7 \\
\hline $222.4^{\mathrm{c}}$ & -2.3 & 0.2 & 0.2 & 0.2 & -2.7 \\
\hline $222.4^{\mathrm{c}}$ & 0.6 & 0.4 & 0.6 & 0.4 & 0.4 \\
\hline 240.6 & 0.09 & 0.1 & 0.01 & 0.09 & 0.09 \\
\hline $240.7^{c}$ & 2.4 & 0.4 & 0.2 & -1.2 & 2.4 \\
\hline 475.9 & -0.1 & -0.4 & -0.7 & -0.1 & -0.1 \\
\hline
\end{tabular}

a. \% Error of found $\mathrm{Ag}^{+}$against taken.

b. Taken $\mathrm{Ag}^{+}$in $\mu \mathrm{mol}$.

c. Run in unequal size of titrant drops.

lence point. No essential difference was found. The titrant does not necessarily have to be added in drops, though several drops may be successively added under control of the program. The equivalence points obtained using the five methods were very close to each other, though the last three methods are more recommended on account of the on-line speed of a decision (Table 1).

In estimating the equivalence point from the titration curve, the effect of different drop sizes of titrant on the accuracy of the result was examined in model cases. An aliquot portion ranging from 0.05 to $0.20 \mathrm{~cm}^{3}$ was assumed as a titrant drop in every addition. When a small aliquot of equal size was added as every titrant drops, the most accurate equivalence point was obtained as usual. The larger the size, the larger is the error if drop sizes were not equal. Small sizes of drops gave acceptable errors, but the larger sizes did not. In the present work, however, the use of unequal drop sizes was preferred so as to diminish the restrictions on the experimental conditions. It is required for calculating the equivalence point that the size of titrant drops does not affect the estimation. Errors of the equivalence points estimated by the 4 methods are shown in Table 3. Even when unequal sizes of the titrant drops were used, good results were obtained in Gran's plot 
Table 4 Titrant volume required for anticipation of the equivalence point by Gran's plot method

\begin{tabular}{|c|c|c|c|c|c|c|c|c|c|}
\hline \multirow{2}{*}{ Taken $\mathrm{Ag}^{+} / \mu \mathrm{mol}$} & \multirow{2}{*}{ Threshold range, $\%$} & \multicolumn{2}{|c|}{ B1 } & \multicolumn{2}{|c|}{ B3 } & \multicolumn{2}{|c|}{ B5 } & \multicolumn{2}{|c|}{$\mathrm{Cl}^{\mathrm{b}}$} \\
\hline & & Volume & Error & Volume & Error & Volume & Error & Volume & Error \\
\hline \multirow[t]{3}{*}{116.9} & 1 & 89.5 & -0.4 & 91.8 & -0.5 & 82.4 & 1.8 & & \\
\hline & 0.5 & 91.8 & -0.3 & 91.8 & -0.5 & 98.9 & 0.6 & & \\
\hline & & & & & & & & 108.6 & 0.08 \\
\hline \multirow[t]{4}{*}{240.6} & 1 & 91.5 & -0.3 & 89.2 & 0.01 & 86.9 & 0.02 & & \\
\hline & 0.5 & 93.8 & 0.3 & 89.2 & 0.01 & 89.2 & -0.2 & & \\
\hline & 0.25 & 96.1 & 0.2 & 89.2 & 0.01 & 89.2 & -0.2 & & \\
\hline & & & & & & & & 104.2 & 0.2 \\
\hline \multirow[t]{4}{*}{475.9} & 1 & 87.9 & -1.2 & 89.0 & -1.0 & 87.9 & -0.8 & & \\
\hline & 0.5 & 90.2 & -0.5 & 89.0 & -1.0 & 87.9 & -0.8 & & \\
\hline & 0.25 & 90.2 & -0.5 & 91.4 & -0.7 & 96.0 & 0.6 & & \\
\hline & & & & & & & & 102.1 & -0.1 \\
\hline
\end{tabular}

a. Shown in \% against volume in equivalence point. b. Evaluation by Newtonian difference (2nd derivative) method. Error indicated is obtained from data judged in B5.

method (C2) and the method of least squares (C3), in contrast to the 2nd derivative method $(\mathrm{Cl})$ and the Lagrange's interpolation method (C4).

In potentiometric titrations involving the Nernstian expression, the Gran's plot mehod (C2) is applicable to the estimation of the equivalence point. Though in the Newtonian 2nd derivative method (C1) the titrant should be added over the equivalence point, in Gran's plot method, the equivalence point may be anticipated on-line before it is attained. The straight line of Eq. (2) was calculated after determining $E$ by a linear method of least squares by including the latest set of $E$ and $\nu$. The point, at which the calculated line crosses the axis of the abscissa, converged step by step. The certainty of anticipating the equivalence point increases. There existed an optimal number of points for the Gran's plot calculation using the method of least squares, since irregular data affected the long-range results. The use of 6 or 8 points seemed to be best in the threshold range from 1 to $0.25 \%$. The titrant volume necessary for anticipation of the equivalence point is shown in Table 4 in percentages against the equivalence quantities. Silver ions, estimated as the mean of several runs, are also indicated in Table 4 in relative error of the found value to the taken. In the last column, equivalence point estimated by $\mathrm{Cl}$ and its error were shown for a comparison. This means, therefore, that titrant addition could be completed before reaching the equivalence point so as to save titration time and titrant reagent (about $10 \%$ or more).

In C3, the observed titration curve was also fitted to a 3rd-order expression, $y=a x^{3}+b x^{2}+c x+d$, by the method of least squares and a Gaussian elimination technique. Errors became smaller when equal numbers of data points, expected from the first derivatives immediately before and after the equivalence point, were used for the calculation. Data points obtained only before the equivalence point were not sufficient to afford a reliable result, even if points near the equivalence point were used. The use of data far from the equivalence point was rather disadvantageous.

For a general data-analysis method, the Lagrange's interpolation method (C4) was examined for estimating the equivalence point. Four sets of $E$ and $v$ were used for the calculation, errors shown in Table 3 being almost of the same magnitude as those obtained by $\mathrm{Cl}$.

In this work, the automated potentiometric precipitation titration in combined use of the Gran's plot method was recommended for an earlier, more accurate anticipation of the equivalence point.

\section{References}

1. S. Fujieda and N. Nishi, Analyst [London], 112, 1185 (1987).

2. G. Gran, Acta Chem. Scand., 4, 559 (1950); Analyst [London], 77, 661 (1952).

3. R. D. Braun, "Introduction to Instrumental Analysis", Chap. 22, McGraw-Hill Book Co., New York, 1987.

(Received February 6, 1989)

(Accepted April 17, 1989) 